Artigo de ReVISÃo

REVIEW ARTICLE

\title{
O Uso de Drogas Anti-Reumáticas na Gravidez ${ }^{*}$ )
}

\section{Use of Anti-Rheumatic Drugs during Presnancy}

\author{
Roger A. Levy ${ }^{(1)}$
}

\section{RESUMO}

A prescrição de drogas anti-reumáticas em pacientes férteis deve ter em conta o conhecimento atual sobre os efeitos na concepção, gestação e lactação. Um aconselhamento que levasse em consideração os riscos e benefícios do planejamento da gravidez, quando possível, seria o ideal. Com a incorporação de novas substâncias e o aparecimento constante de novos dados na literatura, esse assunto deve ser continuamente atualizado. $\mathrm{O}$ estagiamento de acordo com o fator de risco estabelecido pelo FDA é algumas vezes contraditório em relação a nossa prática, em parte porque os estudos em modelos animais podem não ser diretamente aplicáveis a humanos. A terapia com agentes modificadores da resposta biológica é aparentemente segura durante a gestação, considerando-se que tais agentes sejam compostos por moléculas grandes, incapazes de atravessar a placenta. Antiinflamatórios não-esteroidais (incluindo os inibidores específicos de COX-2) podem prejudicar a implantação do óvulo, mas o seu uso é possível no caso da gestação estar em curso, devendo ser evitados após 32 semanas do início da gravidez, quando estão relacionados com complicações fetais. Os inibidores específicos de COX-2 devem ser evitados pelo risco de malformação renal. Aspirina em baixa dose pode ser usada seguramente, quando indicada durante a gravidez. Há preferência pelas heparinas de baixo peso molecular, desde que a não fracionada apresente maior risco de induzir trombocitopenia e sangramento. A hidroxicloroquina é usada e de fato recomendada em gestantes com lúpus, com benefícios para a paciente e sem risco fetal. Varfarina é teratogênica se usada da $6^{a}$ a $9^{a}$ semana de gestação, mas pode ser usada durante o segundo e terceiro trimestres até a $34^{a}$ semana, quando deve ser interrompida com o intuito de evitar sangramento fetal. A imunoglobulina endovenosa é recomendada em casos difíceis de síndrome do anticorpo antifosfolipídeo e vem sendo estudada na prevenção do bloqueio cardíaco total da síndrome do lúpus neonatal. O uso de prednisona e prednisolona é limitado a menor dose eficaz, não atinge a circulação fetal, mas pode induzir os efeitos colaterais maternos já conhecidos. Azatioprina e ciclosporina são utilizadas, quando indicadas

\section{ABSTRACT}

The prescription of anti-rheumatic drugs in fertile patients should take into account the current knowledge about their effects on conception, pregnancy and lactation. Judicious advice and pregnancy planning is ideal when possible. With the incorporation of new substances and the constant appearance of recent data in the literature this subject has to be continuously updated. The FDA risk factor rating is sometimes contradictory to our practice, in part because results from animal studies may not be directly applicable to humans. Biologic response modifiers seem to be safely used during pregnancy, since they are large molecules that are not capable of crossing the placenta. Non-steroidal anti-inflammatory drugs including specific COX-2 inhibitors may impair implantation of the ovum but can be used once pregnancy is under way, they should be avoided after 32 weeks, when there is a relationship with fetal complications. COX-2 inhibitors must be avoided due to its risk of renal malformation. Low-dose aspirin can be used safely during pregnancy. Low molecular weight heparins are preferred, since the unfractionated heparins have an increased risk of inducing thrombocytopenia and bleeding. Hydroxychloroquine is used and in fact recommended in lupus pregnancy with patients' benefits and no fetal risk. Warfarin is teratogenic if given between the $6^{\text {th }}$ and $9^{\text {th }}$ gestational weeks, but can be used during the second and third until 34 weeks, when it should be withdrawn in order to avoid the risk of neonatal bleeding. Intravenous immunoglobulin is recommended for difficult antiphospholipid cases and is being studied to prevent the congenital heart block of neonatal lupus. Prednisone and prednisolone use is limited to the least effective dose since they do not reach fetal circulation, but can induce maternal side effects. Azathioprine and cyclosporine are used when necessary with no apparent fetal harm. Methotrexate and leflunomide must be avoided by all means and the treatment

* Trabalho realizado na Disciplina de Reumatologia, Faculdade de Ciências Médicas, Universidade do Estado do Rio de Janeiro (FCM-UERJ), Rio de Janeiro, RJ, Brasil. Recebido em 02/03/2005. Aprovado, após revisão, em 19/05/2005.

1. Professor adjunto de Reumatologia. FCM-UERJ.

Endereço para correspondência: Dr. Roger A. Levy. Hospital Universitário Pedro Ernesto. Blvd 28 de Setembro 77, sala 333, Vila Isabel. Rio de Janeiro, RJ, Brasil. Tel./fax: 55 (21) 2529-8059; e-mail: rlevy@uerj.br 
formalmente, sem aparente risco fetal. Metotrexato e leflunomide devem ser evitados a qualquer custo e o tratamento interrompido três meses antes da tentativa de concepção. Todas as decisões terapêuticas em pacientes grávidas devem ser individualizadas e os riscos e benefícios considerados.

Palavras-chave: gestação, AINEs, prednisona, hidroxicloroquina.

O tratamento das doenças reumáticas em mulheres na idade fértil envolve aconselhamento sobre contracepção e gestação. É crucial que o internista, reumatologista e obstetra estejam a par das indicações, limitações e riscos do crescente arsenal terapêutico utilizado atualmente em reumatologia. Decisões judiciosas devem ser tomadas de acordo com cada caso, e as controvérsias relacionadas à gestação têm a preocupação com o risco fetal. Algumas drogas classificadas como sendo de alto risco, com base em estudos com modelos animais, são utilizadas durante a gestação em humanos para controlar a doença materna em atividade ${ }^{(1)}$. A identificação de que a exposição in utero ao dietil-stilbesterol poderia resultar em adenocarcinoma da vagina vinte anos depois, deve servir de lição para nos lembrar que os efeitos a longo prazo de alguns agentes seriam somente reconhecidos no futuro.

Até a década de 1980, o aconselhamento mais comum às mulheres com doenças reumáticas, especialmente lúpus eritematoso sistêmico (LES), esclerose sistêmica e síndromes vasculíticas, era o de evitar a gravidez por causa da percepção de risco materno e fetal de uma magnitude inaceitável. Atualmente parece haver um consenso de que a gestação deva ser adiada até que a atividade da doença seja controlada, sendo que certos medicamentos apropriados (como a hidroxicloroquina [HCQ] para o LES) devem ser continuados para reduzir a chance de ativação durante a gestação. A maioria dos dados sobre gestação em condições reumáticas auto-imunes é originária de análises retrospectivas, mas alguns estudos prospectivos envolvendo o uso de drogas anti-reumáticas foram realizados nas duas últimas décadas. No entanto, esse campo permanece com poucos estudos clínicos randomizados ${ }^{(2)}$. Mulheres que usam drogas citotóxicas devem ser informadas dos riscos de prejuízo da fertilidade e de malformações congênitas, e devem usar métodos eficazes de contracepção. Durante a gestação, os antiinflamatórios não esteroidais (AINEs) podem ser utilizados até seis semanas antes da data provável do parto. Doses baixas ou moderadas de corticoesteróides são relativamente seguras durante toda a gestação. Os corticosteróides são freqüente- stopped three months before conception. Every therapeutic decision for a pregnant patient has to be individualized and the risk and benefits considered.

Keywords: pregnancy, NSAIDs, prednisone, hydroxychloroquine.

mente necessários no controle da atividade de várias doenças reumáticas e com o intuito de impedir danos orgânicos sérios. Dentre os chamados agentes modificadores de doença, os tratamentos com sulfasalazina e HCQ podem ser continuados durante a gestação. Drogas citotóxicas podem ser utilizadas após o primeiro trimestre no tratamento de complicações com risco de vida. Ciclosporina e azatioprina podem ser administradas por toda a gestação, se for necessário para o controle da doença. Não dispomos de dados sobre o risco de usar o micofenolato mofetil durante a gestação. Os estudos animais com esse agente revelam aumento de teratogenicidade, e pelo menos um relato de teratogenicidade humana é conhecido. Nos Estados Unidos da América, o micofenolato mofetil não pode ser prescrito em gestantes. Anticorpos monoclonais, embora sejam anticorpos, não tem capacidade de cruzar a barreira placentária. A gravidade da doença a ser tratada influencia na decisão se o uso de uma dessas substâncias é justificado. A retirada profilática quando se planeja uma gestação é mandatória em pacientes em uso de leflunomida e de agentes citotóxicos, com efeito teratogênico conhecido, como metotrexato e ciclofosfamida ${ }^{(3)}$. Durante o aleitamento, prednisona, sulfasalazina e HCQ podem ser utilizados com benefício. Mulheres usando heparina na prevenção de complicações da síndrome do anticorpo antifosfolipídeo durante a gravidez devem tomar as medidas para prevenção de perda de massa óssea e utilizar suplemento de cálcio e vitamina $\mathrm{D}^{(4)}$.

Uma enquete conduzida por Østensen em $2004^{(3)}$ revisou os efeitos colaterais maternos e fetais dos AINEs e agentes imunossupressores em grávidas. Concluiu-se que os AINEs tradicionais não são teratogênicos, mas se administrados tardiamente na gestação podem induzir efeitos deletérios renais e cardíacos. Efeitos semelhantes podem ser esperados com os inibidores específicos de COX-2. AINEs, quando utilizados, devem ser interrompidos com 32 semanas de gestação.

O uso de drogas anti-reumáticas durante a gravidez deve levar em conta os seus efeitos na atividade da doença, na 
gravidez e adversos maternos, assim como no desenvolvimento fetal, no potencial teratogênico, no aleitamento e tardios potenciais na prole. Apresentamos, a seguir, uma revisão das recomendações de indicações e limitações das drogas mais comumente utilizadas em reumatologia. Adotamos a ordem dos fatores de risco proposta pelo Food and Drug Administration em 1980 e revisada em 2002 ${ }^{(1)}$, descritos na Tabela 1. Essa classificação designa as categorias de A a D, e X com indicações de asterisco $\left(^{*}\right.$ ) (quando a informação da literatura diverge daquela contida na bula fornecida pelo fabricante) ou ${ }_{M}$ (quando a informação da classificação tem como base a bula fornecida pelo fabricante).

$\mathrm{Na}$ categoria A constam apenas algumas vitaminas, como o ácido fólico, que é de fato recomendado na dose de $5 \mathrm{mg} / \mathrm{d}$, se possível antes da concepção até pelo menos o final do primeiro trimestre de gestação. O cloreto de potássio é também uma das poucas sustâncias classificadas na categoria A e recomendamos em pacientes que necessitam de corticoesteróides em doses acima de $20 \mathrm{mg} / \mathrm{d}$.

1. Drogas com fator de risco B são aquelas em que não se demonstrou um risco em modelos animais ou nenhum risco em fetos humanos foi comprovado, e nenhum dado de risco em estudo controlado em humanos foi publicado ou nenhum estudo com defeitos em animais ou humanos foi confirmado no primeiro trimestre ou mais tarde.

1.1 Acetaminofeno ou paracetamol (B) tem sido utilizado com segurança em todos os estágios da gestação; doses altas continuadas devem ser evitadas pelo risco de toxicidade hepática materna. É o antipirético mais utilizado antes do parto em casos de corioamnionite. Um estudo investigou a prevalência de anomalias congênitas e alterações de crescimento em 123 mulheres expostas ao paracetamol durante a gravidez. O grupo controle foi composto de 13.329 gestantes que não utilizaram nenhuma medicação. Os autores não encontraram um maior risco de teratogenia e nenhuma influência do paracetamol no crescimento fetal ${ }^{(5)}$. O aleitamento em uso de paracetamol é seguro ${ }^{(6)}$.

1.2 Aspirina infantil (AAS) - de 75 a 100mg/dia, é muito utilizada na gestação como agente anti-agregante plaquetário, para prevenir pré-eclâmpsia e complicações trombóticas da síndrome do anticorpo antifosfolipídeo (SAF). A administração de aspirina infantil durante a gestação não aumenta o risco de sangramento neonatal nem materno e seu uso é adotado nos principais centros de referência ${ }^{(7)}$. Não há risco demonstrado de teratogenicidade. Alguns

TABELA 1

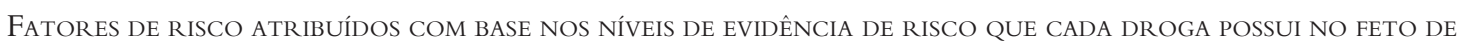
ACORdo COM ESTUdOS EM ANIMAIS E HUMANOS (MODIFICADA DA REFERÊNCIA 1)

\begin{tabular}{|c|c|c|}
\hline Categoria & Justificativa & Exemplo \\
\hline A & $\begin{array}{l}\text { Estudos controlados sem evidência de risco fetal no } \\
1^{0} \text { trimestre ou depois }\end{array}$ & Ácido fólico, vitamina D \\
\hline B & $\begin{array}{l}\text { Estudos animais sem risco fetal sem estudos controlado } \\
\text { s em humanos } 0 U \text { estudos animais com risco que não foi } \\
\text { comprovado em humanos no } 1^{\circ} \text { trimestre (sem evidência } \\
\text { de risco mais tardiamente) }\end{array}$ & $\begin{array}{l}\text { Acetaminofeno, aspirina infantil, sulfasalazina, } \\
\text { agentes anti-TNF, ciclobenzaprina }\end{array}$ \\
\hline C & $\begin{array}{l}\text { Estudos em animais revelaram efeitos teratogênicos e não } \\
\text { existem estudos controlados em humanos, ou estudos em } \\
\text { animais e humanos não estão disponíveis. São substâncias } \\
\text { que só devem ser utilizadas se os benefícios potenciais } \\
\text { justificarem os potenciais riscos para o feto }\end{array}$ & $\begin{array}{l}\text { Certos AINHs/D, inibidores de COX-2/D, prednisona, } \\
\text { prednisolona* } / D \text {, sais de ouro, hidroxicloroquina, } \\
\text { micofenolato mofetil, ciclosporina, GGEV, cevimelina, } \\
\text { pilocarpina, amitriptilina, tramadol, gabapentina }\end{array}$ \\
\hline D & $\begin{array}{l}\text { Evidência positiva de risco humano fetal, usada se os } \\
\text { benefícios forem consideráveis apesar do risco (risco de vida) }\end{array}$ & $\begin{array}{l}\text { Azatioprina, d-Penicilamina, AINEs ( } 3^{\circ} \text { trimestre), } \\
\text { ciclofosfamida, colchicina, varfarina }\left(2^{\circ} \text { e } 3^{\circ} \text { trimestres }\right)\end{array}$ \\
\hline$x$ & $\begin{array}{l}\text { Anomalias fetais em estudos com animais e humanos. } 0 \text { uso } \\
\text { não justifica qualquer benefício possível. Contra-indicado em } \\
\text { mulheres com possibilidade de se tornarem grávidas }\end{array}$ & $\begin{array}{l}\text { Talidomida, metotrexato, leflunomida, misoprostol, } \\
\text { bosentana, bisfosfonatos (?) }\end{array}$ \\
\hline M & Produto classificado pelo fabricante na bula & \\
\hline * & Divergência na classificação do fabricante e de autores independentes & Azatioprina \\
\hline
\end{tabular}


autores sugerem a suspensão da aspirina uma a duas semanas antes do parto para reduzir a possibilidade de sangramento uterino, ou resultante de lacerações vaginais ou cervicais, mas nem todos seguem essa recomendação e não parece haver grandes problemas com o uso continuado. Aspirina em dose antiinflamatória é classificada como (C).

1.3 Os dados sobre uso de sulfasalazina $\left(\mathrm{B}_{\mathrm{M}}{ }^{*}\right)$ em gestantes são quase que totalmente derivados de estudos e relatos em pacientes com doenças inflamatórias intestinais. O uso próximo ao termo é relacionado com icterícia prolongada do neonato e deve ser evitado; por conta disso o seu uso no terceiro trimestre é classificado com risco (D). A dose máxima recomendada é de $2 \mathrm{~g}$ /dia e o uso concomitante de ácido fólico (A) é indicado. Por agir como um inibidor do folato, a sulfasalazina tem uma ação teratogênica em potencial. Norgard et al. realizaram um estudo caso controle com o banco de dados do Hungarian Case Control Surveillance of Congenital Abnormalities, de 1980 a 1996, com 22.865 neonatos ou anomalias fetais congênitas e 38.151 sem anomalias congênitas (grupo controle). O porcentual de mulheres expostas a sulfasalazina durante a gestação foi igual nos dois grupos (0,07\%). Nenhuma dessas análises indica qualquer elevação significativa na prevalência de anomalias congênitas em comparação a pacientes não expostas ${ }^{(8)}$. O aleitamento é permitido em uso de sulfasalazina ${ }^{(6)}$.

1.4 Ciclobenzaprina $\left(\mathrm{B}_{\mathrm{M}}{ }^{*}\right)$ e sertralina $\left(\mathrm{B}_{\mathrm{M}}{ }^{*}\right)$ são considerados os agentes mais seguros para o tratamento da fibromialgia durante a gestação, embora existam poucos dados.

1.5 Heparinas de baixo peso molecular (HBPM) $\left(\mathrm{B}_{\mathrm{M}}{ }^{*}\right)$ como enoxaparina e dalteparina têm sido amplamente utilizadas na profilaxia de trombose em gestantes com SAF. Embora as HPBM tenham um índice terapêutico excelente e um risco menor de indução de trombocitopenia do que as heparinas não fracionadas ( $\mathrm{HNF})\left(\mathrm{C}_{\mathrm{M}}\right)$, o uso por longo prazo de HBPM, assim como das HNF, pode estar associado com maior perda de massa óssea durante a gravidez. Por isso recomenda-se suplementação com $1.000 \mathrm{mg}$ de cálcio e 400 UI de vitamina D $\left(\mathrm{A}^{*}\right)$. O aleitamento é permitido em mulheres em uso de HBPM, desde que essas sejam digeridas pelo lactente; as HNF não passam pelo leite.

1.6 Os dados em humanos são limitados com o uso de anticorpos monoclonais anti-TNF (infliximabe [B] e adalimumabe) e anti-CD20 (rituximabe [C]), e com proteínas de fusão anti-TNF como o etanercepte $\left(\mathrm{B}_{\mathrm{M}}\right)$ durante a gestação. Essas substâncias são proteínas grandes e não cruzam a placenta, e se o fazem, aparentemente não alcançam a circulação fetal. A melhor informação disponível vem de relatos do uso de infliximabe em gestantes. Uma série descreveu 96 mulheres com doença de Chron ou artrite reumatóide, inclusive algumas com exposição durante o primeiro trimestre. Os autores concluíram que o infliximabe não foi associado com complicações da gestação $\mathrm{O}^{(9)}$. Existe um relato de um caso de uso acidental de rituximabe durante o primeiro trimestre de gestação em uma paciente com linfoma não-Hodgkin. A gestação foi normal, e uma criança saudável nasceu a termo. Estudos hematológicos e imunológicos não revelaram alterações ${ }^{(10)}$.

Os limitados dados atuais sugerem que os antagonistas de TNF não têm ação embriotóxica ou teratogênica ${ }^{(11)}$.

Sills et al. descreveram o primeiro caso de indução de ovulação, inseminação intra-uterina, gestação normal e nascimento de uma criança saudável seguidos de mais de um ano de uso de anti-TNF para artrite reumatóide ${ }^{(12)}$. Não se recomenda iniciar a terapia com anti-TNF durante a gestação. Anticorpos monoclonais e proteínas de fusão são excretados no leite, mas são digeridos pelo suco gástrico do lactente.

2. Drogas com fator de risco C são aquelas cujos estudos em animais revelaram efeitos teratogênicos, não existindo estudos controlados em humanos, ou não estando disponíveis estudos em animais e humanos. Seu uso deve ser judicioso quando os benefícios são maiores do que os riscos potenciais.

2.1 A maioria dos antiinflamatórios não esteroidais (AINEs) é classificada como (C), mas não devem ser utilizados no terceiro trimestre (quando são classificados com o fator de risco [D]) por causarem constrição do ducto arterioso fetal que pode acarretar em hipertensão arterial pulmonar. Com menor freqüência, perdas fetais, baixo peso ao nascer e alterações glomerulares e de coagulação foram relatadas. Mesmo assim, alguns pacientes necessitam de tratamento com antiinflamatório, casos em que damos preferência a prednisona em baixas doses. Indometacina $\left(\mathrm{B}^{*}\right)$ é amplamente utilizada na prevenção do parto prematuro, mas tem sido relacionada a casos de enterocolite ${ }^{(13)}$. O uso de ibuprofeno $\left(\mathrm{B}_{\mathrm{M}}{ }^{*}\right)$ foi relacionado a casos de defeitos glomerulares acarretando insuficiência renal ${ }^{(1)}$. A influência do uso de AINEs e a concepção foram revisadas no cadastro dinamarquês envolvendo 1.462 gestantes que usaram AINEs (excluindo a indometacina) em um período de 30 dias antes da concepção até o nascimento comparado com 17.259 gestantes que não utilizaram nenhum medicamento durante a gestação. A análise dos dados foi feita para estimar o risco de resultados gestacionais adversos. 
Os autores concluíram que o uso de AINEs durante a gestação não parece aumentar o risco de resultados gestacionais adversos, mas é associado com maior risco de abortamento $^{(14)}$

Um estudo populacional de uma coorte sobre o uso pré-natal de AINEs, aspirina, e acetaminofeno com entrevistas pelo programa de assistência Kaiser Permanente, em São Francisco, CA, EUA, envolvendo 1.055 mulheres, avaliou os resultados até 20 semanas de gestação. O uso de AINEs foi associado com um aumento de $80 \%$ no risco de aborto. Essa associação foi maior quando o AINE foi utilizado próximo à concepção. Já o uso de acetaminofeno, não foi associado com abortamento independente do tempo ou época de uso ${ }^{(15)}$.

O uso de AINEs com segurança durante a gestação é apoiado por um estudo prospectivo que envolveu 88 gestantes com doenças reumáticas inflamatórias divididas em dois grupos: 45 que utilizaram e 43 que não utilizaram AINEs durante a gestação. Possíveis efeitos a longo prazo no desenvolvimento da criança foram pesquisados por entrevista telefônica. Não foram encontradas diferenças significativas ente os grupos estudados ${ }^{(16)}$. O aleitamento é permitido por mulheres em uso de AINEs. Embora esses sejam excretados em pequenas quantidades no leite materno, nenhuma toxicidade foi relatada ${ }^{(6)}$.

2.2 O inibidor seletivo de COX-2 celecoxibe $\left(\mathrm{C}_{\mathrm{M}}{ }^{*}\right)$ foi introduzido no mercado no final de 1998 e por isso dispomos de poucos estudos e relatos em gestantes. Perdas pré e pós implantacionais foram evidentes com celecoxibe da mesma forma que foram com indometacina em ratos. O mecanismo de interferência na implantação, tanto de AINEs clássicos (não seletivos) como de inibidores específicos de COX-2, parece ser por defeito de decidualização. Portanto, os inibidores específicos de COX2 devem ser utilizados com cautela em mulheres que planejam engravidar. De fato, foi sugerido que os inibidores específicos de COX-2, em razão de seu perfil de segurança gástrica, tenham um papel potencial na contracepção póscoito $^{(17)}$. Ambas as substâncias, celecoxibe e valdecoxibe (e a pró-droga parenteral parecoxibe), são indicadas no tratamento da dismenorréia primária, e dor pós-operatória, incluindo histerectomia, e dor associada a endometriose. Essas substâncias também são tocolíticos eficazes sem os riscos gástricos dos AINEs não específicos ${ }^{(18)}$. As recomendações em relação a concepção são as mesmas dos AINEs não específicos ${ }^{(19)}$. Assim como os AINEs não específicos, os inibidores específicos de COX-2 não devem ser utilizados no terceiro trimestre (D) pelo risco de fechamento prematuro do ducto arterioso ${ }^{(20)}$. O aleitamento deve ser evitado em pacientes usando celecoxibe ou valdecoxibe.

2.3 Ao contrário dos corticóides fluorados, prednisona $\left(\mathrm{C}^{*}\right)$ e prednisolona $\left(\mathrm{C}^{*}-\mathrm{D}\right.$ no primeiro trimestre) são inativadas na placenta, com menos de $10 \%$ da droga ativa detectada na circulação fetal. No entanto, o uso desses corticóides durante a gestação é relacionado de forma dose dependente com vários efeitos adversos maternos, incluindo diabetes, edema, hipertensão arterial, pré-eclâmpsia, ruptura prematura de membranas, imunossupressão, osteopenia e osteonecrose. Prednisona e prednisolona são indicadas durante a gestação de pacientes com LES no controle de artrite, alveolite, pericardite e miosite. A suplementação de cálcio e vitamina $\mathrm{D}$ é aconselhável nas pacientes expostas por mais de duas semanas ${ }^{(1)}$.

O serviço de informação israelense sobre teratogenia analisou prospectivamente 311 gestações expostas a corticóides durante o primeiro trimestre em função da freqüência de anomalias congênitas em comparação a 790 controles. Os resultados do estudo sugerem que os corticóides não possuem risco de teratogenia em humanos ${ }^{(21)}$. Recomenda-se o uso parcimonioso em pacientes com LES ou dermatomiosite durante a gestação, quando são necessárias doses moderadas ou elevadas por longos períodos e a HCQ já esteja sendo utilizada; o uso de azatioprina pode ser considerado.

Existem vários protocolos adotados para a proteção adrenal durante intervenções, como parto normal ou cesáreo em pacientes em uso corrente ou que utilizaram corticoesteróides até os últimos dois anos. Em nosso Centro utilizamos hidrocortisona $100 \mathrm{mg} 8 / 8$ horas por três dias. O aleitamento não é contra-indicado em pacientes usando até $40 \mathrm{mg} /$ dia de prednisona ou prednisolona.

2.4 Os corticoesteróides fluorados, como dexametasona e betametasona $\left(\mathrm{C}^{*}\right)$, são usados na gestação para acelerar a maturidade pulmonar em gestações com risco de parto prematuro. A betametasona é preferencialmente utilizada quando o bloqueio cardíaco congênito da síndrome do lúpus neonatal é diagnosticado ${ }^{(22)}$.

2.5 Sais de ouro $\left(\mathrm{C}_{\mathrm{M}}\right)$ não foram relacionados com indução de teratogenia na gestação humana, embora alterações de parede abdominal e defeitos de crescimento tenham sido observados em roedores. Embora a freqüência do seu uso esteja caindo, a paciente em uso de sais de ouro não precisa interromper o tratamento durante a gestação, mas não se recomenda iniciar o tratamento durante a gestação ${ }^{(23)}$. $\mathrm{O}$ aleitamento é permitido em uso de sais de ouro ${ }^{(6)}$. 
2.6 No passado havia preocupações com relação aos efeitos dos antimaláricos durante a gestação ${ }^{(24)}$; essas foram se reduzindo com base nas análises recentes. Atualmente, a HCQ (C) não é interrompida na gestação de pacientes com LES. Não somente pelo longo tempo de eliminação, mas a interrupção dos antimaláricos pode precipitar crises de LES $^{(25)}$. Em adultos, o uso crônico de cloroquina, e em menor grau o de HCQ, estão relacionados a um pequeno risco de retinopatia pigmentar que pode ameaçar a visão. Para obter os dados de segurança na gestação, foi examinada a função visual de 21 crianças nascidas de mulheres que utilizaram antimaláricos durante a gestação. Nenhuma anomalia foi detectada ${ }^{(26)}$. Outros estudos recentes em pacientes com LES tratados com antimaláricos também não mostraram efeitos adversos na gestação nem no feto ${ }^{(27)}$. Um estudo controlado randomizado avaliou a segurança do uso de HCQ durante a gestação de 20 pacientes com LES ou lúpus discóide. Nenhum dos pacientes no grupo da HCQ apresentou exacerbação do LES. Pré-eclâmpsia foi diagnosticada em três pacientes do grupo placebo (uma com morte fetal). A dosagem média de prednisona foi menor no grupo da HCQ. A idade gestacional e o índice de Apgar foi maior no grupo tratado com a HCQ. O exame neonatal não revelou anomalias oculares, neurológicas nem auditivas após 1,5 a $3 \operatorname{anos}^{(28)}$. Setenta e oito médicos experientes no tratamento do LES responderam a um questionário elaborado sobre sua experiência com uso de antimaláricos em gestação e aleitamento. Dos 67\% que responderam, nenhum relatou ter visto anomalias fetais. Após o parto, $63 \%$ continuam o tratamento com aleitamento. A maioria mantém o tratamento com HCQ durante a gestação ${ }^{(29)}$.

Uma série de 133 gestações em mulheres tratadas com HCQ, resultante em 117 nascidos vivos, foi comparada com um grupo controle de 53 mulheres que não receberam HCQ. O índice de sucesso gestacional foi semelhante nos dois grupos. O estudo eletrocardiográfico não revelou diferenças e não foram detectadas anomalias visuais, auditivas, de crescimento ou de desenvolvimento. Os autores sugerem que a terapia com a HCQ deve ser mantida por toda a gestação em pacientes com $\operatorname{LES}^{(30)}$. O aleitamento é permitido em usuários de antimaláricos ${ }^{(6)}$.

$2.7 \mathrm{O}$ micofenolato mofetil (MMF) $\left(\mathrm{C}_{\mathrm{M}}\right)$ induz reabsorção fetal e malformações em ratos e coelhos na dose humana máxima recomendada. O MMF provavelmente cruza a placenta e se recomenda que a paciente interrompa o tratamento seis semanas antes de tentar conceber. Foi relatado um caso de múltiplas malformações em um filho de uma paciente tratada com MMF antes de engravidar e durante o primeiro trimestre da gravidez ${ }^{(31)}$. Por outro lado, outro caso de gestação bem sucedida foi relatado em uma paciente exposta ao MMF no primeiro trimestre de gestação ${ }^{(32)}$. Aleitamento em pacientes usando MMF não é aconselhável em decorrência do risco aumentado de infecções e linfoma ${ }^{(6)}$.

2.8 Ciclosporina $\left(\mathrm{C}_{\mathrm{M}}\right)$ é um imunossupressor usado em várias situações clínicas em reumatologia. Seu uso foi relacionado com embriotoxicidade em camundongos expostos a 2,5 vezes a dose humana máxima recomendada em humanos. A ciclosporina foi ligada com baixo peso ao nascer, diabetes gestacional e hipertensão arterial. A maioria dos dados deriva de estudos em pacientes transplantadas renais; de qualquer forma, os efeitos imunes a longo prazo são desconhecidos ${ }^{(33)}$. Aleitamento não é recomendado em pacientes em uso de ciclosporina; leucopenia dentre outras alterações foi relatada ${ }^{(6)}$.

2.9 A gamaglobulina endovenosa (GGEV) $\left(\mathrm{C}_{\mathrm{M}}\right)$ tem sido utilizada por mais de 20 anos com benefício para pacientes com várias doenças auto-imunes. Relatos em humanos sugerem um ótimo perfil de segurança. Suas principais indicações em reumatologia são na prevenção de complicações da SAF e do bloqueio cardíaco do lúpus neonatal ${ }^{(7)}$. Não dispomos de dados sobre o aleitamento em pacientes em uso de GGEV.

2.10 A administração oral de cevimelina ou pilocarpina $\left(\mathrm{C}_{\mathrm{M}}\right)$ é indicada para a melhora da secura das mucosas que ocorre na síndrome de Sjögren. Seu uso foi relacionado com redução de implantação em ratos com cinco vezes a dose humana máxima recomendada e induziu morte fetal com dez vezes a dose humana máxima recomendada. Ambas as substâncias têm baixo peso molecular e são capazes de cruzar a placenta ${ }^{(1)}$. A excreção no leite parece ocorrer em ambas; o seu uso, portanto, deve ser evitado no aleitamento $^{(6)}$.

3. Drogas classificadas com risco D possuem evidência positiva de risco fetal humano, mas os benefícios podem ser aceitáveis dependendo do risco (condições com risco de vida).

3.1 O uso de azatioprina $\left(\mathrm{D}_{\mathrm{M}}\right)$ em mulheres férteis utilizando dispositivos intra-uterinos (DIU) é associado com redução da eficácia. Em gestação humana, o seu uso parece ser seguro, desde que o figado fetal não é capaz de converter a azatioprina na sua forma ativa. Parto prematuro e toxicidade medular foram relatados, e restrição do crescimento também pode ocorrer. Uma grande série foi relatada em pacientes com doença inflamatória intestinal ${ }^{(34)}$. Em dosagens de 1,5-2,0 mg/kg/d a azatioprina não foi rela- 
cionada com teratogenia em humanos ${ }^{(35)}$. Ainda se debate sobre o possível impacto do uso de azatioprina e ciclosporina no sistema imune fetal. Em neonatos expostos a essas drogas, os níveis de imunoglobulinas e resposta à vacina da hepatite $\mathrm{B}$ não diferiram de controles ${ }^{(36)}$. $\mathrm{O}$ aleitamento é formalmente contra-indicado em mulheres que usam azatioprina; já foi relacionado com atraso do desenvolvimento sexual e alterações da fertilidade ${ }^{(6)}$.

$3.2 \mathrm{O}$ uso de penicilamina $\left(\mathrm{D}_{\mathrm{M}}\right)$ durante a gestação é associado com um risco de malformações de 5\%. As pacientes contemplando gestação devem ser aconselhadas a interromper o uso de penicilamina. Se a gestação for detectada, a pressão arterial e as funções renais e pulmonares devem ser checadas regularmente ${ }^{(37)}$. Aleitamento não é seguro $^{(6)}$.

3.3 A exposição aos derivados coumarínicos $\left(D^{*}\right)$ no início da gestação é associada com uma síndrome de embriopatia chamada de "síndrome varfarínica fetal". A exposição a tais derivados entre a $6^{a}$ e a $8^{a}$ semanas resulta em uma chance de $10 \%$ a $25 \%$ de manifestação dessa síndrome ${ }^{(1)}$. Em casos selecionados, a varfarina e seus derivados podem ser utilizados durante a gestação no segundo e terceiro trimestres, quando os possíveis benefícios são maiores que os riscos ${ }^{(7)}$. A metanálise envolvendo 208 gestações expostas no segundo e terceiro trimestres encontrou $84 \%$ de filhos normais e nenhum caso de "síndrome varfarínica fetal". A exposição próxima ao termo resulta em sangramento neonatal e deve ser evitada. A varfarina é compatível com aleitamento ${ }^{(6)}$. Em nosso centro utilizamos com sucesso e sem efeitos adversos a varfarina da gravidez entre a $14^{\mathrm{a}}$ e a $36^{\mathrm{a}}$ semanas de gestação.

3.4 O uso de ciclofosfamida $\left(D_{M}\right)$ em mulheres férteis é relacionado a uma redução da fertilidade por insuficiência ovariana. O uso de ciclofosfamida durante o primeiro trimestre de gestação é relacionado a múltiplas anomalias fetais e freqüentemente acarreta abortamentos ${ }^{(38)}$. O uso no segundo e terceiro trimestres só deve ser cogitado em casos de risco de vida por doença renal. Aleitamento não é recomendado, porque a ciclofosfamida é excretada no leite e foi relacionada com neutropenia neonatal, supressão imune e carcinogênese.

4. Drogas com risco $\mathrm{X}$ são aquelas relacionadas com efeitos teratogênicos em animais e em humanos. $O$ uso desses agentes não é justificado, e estão formalmente contraindicados em mulheres com possibilidade de engravidar. A talidomida é um exemplo clássico de droga desse grupo. Outra substância que está classificada como X é a bosentana, o inibidor de endotelina, indicado no controle da hipertensão arterial pulmonar.

4.1 Metotrexato (MTX) e leflunomida (X) são inibidores do ácido fólico, suprimem a proliferação linfocitária, e são classificados como inibidores de purina e de pirimidina. $\mathrm{O}$ ácido fólico é essencial para o desenvolvimento embriônico. Ambas as substâncias, MTX e leflunomida, induziram defeitos congênitos em modelos animais e devem ser evitados na gestação humana. Leflunomida apresenta uma teratogenicidade dose dependente com doses equivalentes às utilizadas em humanos. Até mesmo a exposição ocupacional ao MTX pode estar relacionada a risco fetal ${ }^{(39)}$.

Mulheres em uso recente ou corrente de leflunomida e que se descobrem grávidas, devem ser tratadas com colestiramina (B) (8 g 8/8 horas por 11 dias), até que não se detecte mais a leflunomida no plasma. Se o nível de leflunomida é $>0,02 \mathrm{mg} / \mathrm{mL}$ em duas ocasiões com 14 dias de intervalo, a terapia com colestiramina deve ser repetida. $\mathrm{O}$ fabricante recomenda o relato de casos de gestantes em uso de leflunomida com o intuito de monitorar os resultados. Esse programa de vigilância dispõe de atendimento telefônico nos EUA (1-877-311-8972). Ambas as substâncias, MTX e leflunomida, são contra-indicadas se a gestação for planejada; em decorrência de sua meia-vida longa, a leflunomida deve ser suspensa por pelo menos dois meses previamente à tentativa de se engravidar.

Uma enquete foi respondida por 175 reumatologistas sobre sua percepção quanto ao risco fetal e recomendações em relação à contracepção em mulheres férteis em uso de drogas modificadoras da evolução da artrite: a gestação foi contra-indicada em mulheres usando MTX (95\%) ou leflunomida (92,7\%). A série relatou 38 casos de gestação durante a terapia com MTX e 10 com leflunomida: três das que usaram MTX tiveram malformações congênitas ${ }^{(40)}$. Nos EUA, quando uma mulher em uso de MTX se descobre grávida, o aborto é indicado; se a paciente se recusa, recomenda-se a suplementação com $5 \mathrm{mg} / \mathrm{d}$ ou mais de ácido fólico, e monitoramento com ultra-sonografia quinzenal. Em nossa experiência, os casos com malformações grosseiras acabam em abortamento espontâneo. A Academia Americana de Pediatria recomenda evitar o aleitamento em uso de MTX, em razão de uma série de problemas potenciais, incluindo imunossupressão, neutropenia; assim como prejuízo no crescimento e carcinogênese. Aleitamento não é recomendado em mulheres em uso de leflunomida: seu baixo peso molecular permite sua excreção no leite ${ }^{(6)}$. 
Concluímos que o bom senso e as decisões em equipe devem ser os fundamentos das decisões tomadas no tratamento de gestantes com doenças reumáticas; que o ideal seria planejar a gestação para um período livre de atividade inflamatória e sem medicações que interferem na concepção e na evolução da gestação; e que o reumatologista deve estar constantemente se atualizando sobre os novos estudos em relação a medicamentos e gestação. As Tabelas 2 e 3 são guias resumidos das restrições do uso das principais drogas anti-reumáticas na gravidez e aleitamento.

TABELA 2

EFEITOS DOS ANTIINFLAMATÓRIOS, CORTICOESTERÓIDES E IMUNOSSUPRESSORES NA GESTAC̄̃̃O HUMANA, ALEITAMENTO E REPRODUÇÃO (MODIFICADA DA REFERÊNCIA 41)

\begin{tabular}{|c|c|c|c|c|c|c|c|c|}
\hline Droga & $\begin{array}{l}\text { isco pelo } \\
\text { FDA }\end{array}$ & $\begin{array}{c}\text { Passagem } \\
\text { transplacentária }\end{array}$ & $\begin{array}{c}\text { Teratogenicidade } \\
\text { humana }\end{array}$ & CIUR* & $\begin{array}{l}\text { Efeitos adversos } \\
\text { fetais/neonatais }\end{array}$ & $\begin{array}{l}\text { Presença no } \\
\text { leite }\end{array}$ & $\begin{array}{l}\text { Aleitamento } \\
\text { materno }\end{array}$ & $\begin{array}{l}\text { Redução da } \\
\text { fertilidade }\end{array}$ \\
\hline AINE & $B / D^{\#}$ & Sim & Não & Não & $\begin{array}{l}\text { Constrição do } \\
\text { ducto arterioso, } \\
\text { redução do fluxo } \\
\text { sanguíneo renal }\end{array}$ & Sim & $\begin{array}{l}\text { Possível para } \\
\text { vários }\end{array}$ & $\begin{array}{c}\text { Casos de inibição } \\
\text { de ruptura } \\
\text { do folículo }\end{array}$ \\
\hline Azatioprina & D & Sim & Não & Sim & $\begin{array}{l}\text { Anomalias } \\
\text { congênitas } \\
\text { esporádicas. } \\
\text { Alterações } \\
\text { imunes } \\
\text { transitórias }\end{array}$ & Sim & Não & Não \\
\hline Betametasona & C & Sim & & Sim & Raro & Sem dados & Não & Não foi estudado \\
\hline Ciclofosfamida & D & Sim & Sim & Sim & $\begin{array}{c}\text { Anomalias } \\
\text { cromossômicas, } \\
\text { citopenia }\end{array}$ & Sim & Não & $\begin{array}{l}\text { Em homens } \\
\text { e mulheres }\end{array}$ \\
\hline Ciclosporina & C & Sim & Sim & Sim & $\begin{array}{l}\text { Alterações } \\
\text { imunes } \\
\text { transitórias }\end{array}$ & Sim & Possível (?) & Não \\
\hline $\begin{array}{l}\text { Cloroquina e } \\
\text { Hidroxicloroquina }\end{array}$ & c & Sim & Não & Não & $\begin{array}{l}\text { Não nas doses } \\
\text { recomendadas }\end{array}$ & Sim & Permitido & Não foi estudado \\
\hline Dexametasona & C & Sim & & Sim & $\begin{array}{c}\text { Anomalias } \\
\text { do neuro- } \\
\text { desenvolvimento }\end{array}$ & Sem dados & Não & Não foi estudado \\
\hline $\begin{array}{l}\text { Imunoglobulina } \\
\text { endovenosa }\end{array}$ & C & Sim & Não & Sem dados & Sem dados & Sem dados & Sem dados & Sem dados \\
\hline Leflunomida & $x$ & Sem dados & $\begin{array}{c}\text { Dados } \\
\text { insuficientes }\end{array}$ & $\begin{array}{l}\text { Sem } \\
\text { dados }\end{array}$ & $\begin{array}{c}\text { Dados } \\
\text { insuficientes }\end{array}$ & Sem dados & Não & Não foi estudado \\
\hline Metotrexato & $x$ & Sim & Sim & Sim & citopenia & Sim & Não & $\begin{array}{l}\text { Oligospermia } \\
\text { em altas doses }\end{array}$ \\
\hline $\begin{array}{l}\text { Micofenolato } \\
\text { mofetil }\end{array}$ & C & Sim & $?$ & & $\begin{array}{l}\text { Um relato de } \\
\text { caso com } \\
\text { múltiplas } \\
\text { anomalias }\end{array}$ & Sem dados & Não & Sem dados \\
\hline Prednisona & C & Sim & $\begin{array}{l}\text { Alterações } \\
\text { do palato (?) }\end{array}$ & Sim & Raro & Sim & Permitido & Não foi estudado \\
\hline Sulfasalazina & B & Sim & Não & Não & Nenhuma & Sim & Permitido & Sim, em homens \\
\hline
\end{tabular}

* = Restrição do crescimento intra-uterino; ? = sem comprovação; \# = no terceiro trimestre 
TABELA 3

EFEITOS DAS DROGAS MODIFICADORAS DA RESPOSTA BIOLÓGICA NA GESTAÇÃO, LACTAÇÃO E REPRODUÇ̃̃O HUMANA (MODIFICADA DA REFERÊNCIA 41)

\begin{tabular}{lcccccccc}
\hline Droga & $\begin{array}{c}\text { Risco pelo } \\
\text { FDA }\end{array}$ & $\begin{array}{c}\text { Passagem } \\
\text { transplacentária }\end{array}$ & $\begin{array}{c}\text { Teratogenicidade } \\
\text { humana }\end{array}$ & $\begin{array}{c}\text { CIUR* } \\
\text { Efeitos adversos } \\
\text { fetais/neonatais }\end{array}$ & $\begin{array}{c}\text { Presença no } \\
\text { leite materno }\end{array}$ & $\begin{array}{c}\text { Aleitamento } \\
\text { Redução da } \\
\text { fertilidade }\end{array}$ \\
\hline Adalimumabe & B & Não & Não & Não & Sem dados & Sem dados & Não & Sem dados \\
Anakinra & B & Não & Não & Não & Sem dados & Sem dados & Não & Sem dados \\
Etanercepte & B & Sim & Não & Não & Sem dados & Sim & Não & Sem dados \\
Infliximabe & B & Não & Não & Não & Sem dados & Sim & Não & Não \\
Rituximabe & C & Não & Não & Não & Sem dados & Sem dados & Não & Sem dados \\
\hline
\end{tabular}

* = Restrição do crescimento intra-uterino; ? = sem comprovação

\section{REFERÊNCIAS}

1. Briggs GG, Freeman RK, Yaffe SJ: Drugs in pregnancy and lactation. 6th ed, Lippincott Williams and Wilkins, Philadelphia, PA, 2002.

2. Gordon C: Pregnancy and autoimmune diseases. Best Pract Res Clin Rheumatol 18: 359-67, 2004.

3. Østensen M: Disease specific problems related to drug therapy in pregnancy. Lupus 13: 746-50, 2004.

4. Janssen NM, Genta MS: The effects of immunosuppressive and antiinflammatory medications on fertility, pregnancy, and lactation. Arch Intern Med 160: 610-9, 2000.

5. Thulstrup AM, Sorensen HT, Nielsen GL, et al: Fetal growth and adverse birth outcomes in women receiving prescriptions for acetaminophen during pregnancy. Euro Map Study Group Am J Perinatol 16: 321-6, 1999.

6. [No authors listed]: Committee on Drugs American Academy of Pediatrics. The transfer of drugs and other chemicals into human milk. Pediatrics 93: 137-150, 1994.

7. Tincani A, Branch DW, Levy RA, et al: Treatment of pregnant patients with antiphospholipid syndrome. Lupus 12: 524-9, 2003.

8. Norgard B, Czeizel AE, Rockenbauer M, Olsen J, Sorensen HT: Population-based case control study of the safety of sulfasalazine use during pregnancy. Aliment Pharmacol Ther 15: 483-6, 2001.

9. Katz JA, Antoni C, Keenan GF, Smith DE, Jacobs SJ, Lichtenstein GR: Outcome of pregnancy in women receiving infliximab for the treatment of Crohn's disease and rheumatoid arthritis. Am J Gastroenterol 99: 2385-92, 2004

10. Kimby E, Sverrisdottir A, Elinder G: Safety of rituximab therapy during the first trimester of pregnancy: A case history. Eur J Haematol 72: 292-5, 2004

11. Khanna D, McMahon M, Furst DE: Safety of tumour necrosis factoralpha antagonists. Drug Saf 27: 307-24, 2004.

12. Sills ES, Perloe M, Tucker MJ, Kaplan CR, Palermo GD: Successful ovulation induction, conception, and normal delivery after chronic therapy with etanercept: a recombinant fusion anti-cytokine treatment for rheumatoid arthritis. Am J Reprod Immunol 46: 366-8, 2001.

13. Parilla BV, Grobman WA, Holtzman RB, Thomas HA, Dooley SL: Indomethacin tocolysis and risk of necrotizing enterocolitis. Obstet Gynecol 96: 120-3, 2000.
14. Nielsen GL, Sorensen HT, Larsen H, Pedersen L: Risk of adverse birth outcome and miscarriage in pregnant users of non-steroidal anti-inflammatory drugs: population based observational study and case-control study. Br Med J 322: 266-70, 2001.

15. Li DK, Liu L, Odouli R: Exposure to non-steroidal antiinflammatory drugs during pregnancy and risk of miscarriage: population based cohort study. Br Med J 327(7411): 368, 2003.

16. Østensen M, Østensen H: Safety of nonsteroidal antiinflammatory drugs in pregnant pacientes with rheumatic disease. J Rheumatol 23: 1045-9, 1996.

17. Sookvanichsilp N, Pulbutr P. Anti-implantation effects of indomethacin and celecoxib in rats. Contraception 65: 373-8, 2002.

21. Gur C, Diav-Citrin O, Shechtman S, Arnon J, Ornoy A: Pregnancy outcome after first trimester exposure to corticosteroids: a prospective controlled study. Reprod Toxicol 18: 93-101, 2004.

22. Jobe AH, Soll RF: Choice and dose of corticosteroid for antenatal treatments. Am J Obstet Gynecol 190: 878-81, 2004.

23. Rayburn WF: Connective tissue disorders and pregnancy. Recommendations for prescribing. J Reprod Med 43: 341-9, 1998.

24. Parke AL, Rothfield NF: Antimalarial drugs in pregnancy --the North American experience. Lupus 5 Suppl 1: S67-9, 1996.

25. Borden MB, Parke AL: Antimalarial drugs in systemic lupus erythematosus: use in pregnancy. Drug Saf 24: 1055-63, 2001.

26. Klinger G, Morad Y, Westall CA, et al: Ocular toxicity and antenatal exposure to chloroquine or hydroxychloroquine for rheumatic diseases. Lancet 358(9284):813-4, 2001.

27. Cortés-Hernández J, Ordi-Ros J, Paredes F, Casellas M, Castillo F, Vilardell-Tarres M: Clinical predictors of fetal and maternal outcome in systemic lupus erythematosus: a prospective study of 103 pregnancies. Rheumatology 41: 643-50, 2002.

28. Levy RA, Vilela VS, Cataldo MJ, et al: Hydroxychloroquine (HCQ) in lupus pregnancy: double-blind and placebo-controlled study. Lupus 10: 401-4, 2001.

29. Al-Herz A, Schulzer M, Esdaile JM. Survey of antimalarial use in lupus pregnancy and lactation. J Rheumatol 29: 700-6, 2002.

30. Costedoat-Chalumeau N, Amoura Z, Duhaut P, et al: Safety of hydroxychloroquine in pregnant patients with connective tissue diseases: a study of one hundred thirty-three cases compared with a control group. Arthritis Rheum 48: 3207-11, 2003. 
31. Le Ray C, Coulomb A, Elefant E, Frydman R, Audibert F: Mycophenolate mofetil in pregnancy after renal transplantation: a case of major fetal malformations Obstet Gynecol 103: 1091-4, 2004.

32. Pergola PE, Kancharla A, Riley DJ: Kidney transplantation during the first trimester of pregnancy: immunosuppression with mycophenolate mofetil, tacrolimus, and prednisone. Transplantation 71: 994-7, 2001.

33. Petri M. Immunosuppressive drug use in pregnancy. Autoimmunity 36: 51-6, 2003.

34. Moskovitz DN, Bodian C, Chapman ML, et al: The effect on the fetus of medications used to treat pregnant inflammatory boweldisease patients. Am J Gastroenterol 99: 656-61, 2004.

35. Rajapakse R, Korelitz BI: Inflammatory Bowel Disease During pregnancy. Curr Treat Options Gastroenterol 4: 245-251, 2001.

36. Cimaz R, Meregalli E, Biggioggero M, et al. Alterations in the immune system of children from mothers treated with immunosuppressive agents during pregnancy. Toxicol Lett 149: 155-62, 2004.
37. Lyle WH: Penicillamine in pregnancy. Lancet 1: 606-7, 1998.

38. Matalon ST, Ornoy A, Lishner M: Review of the potential effects of three commonly used antineoplastic and immunosuppressive drugs (cyclophosphamide, azathioprine, doxorubicin on the embryo and placenta). Reprod Toxicol 18: 219-30, 2004.

39. Loyd ME, Carr M, Mcelhatton P, Hall GM, Hughes RA: The effect of methotrexate on pregnancy, fertility and lactation. Q J Med 92: 551-63, 1999.

40. Chakravarty EF, Sanchez-Yamamoto D, Bush TM: The use of disease modifying antirheumatic drugs in women with rheumatoid arthritis of childbearing age: a survey of practice patterns and pregnancy outcomes. J Rheumatol 30: 241-6, 2003.

41. Østensen M, Khamashta MH, Lockshin MD, et al: Antirheumatic drug therapy and reproduction. Arthritis Rheum, submetido para publicação, 2005. 